\title{
Repensar el espacio (rural): entre la individualización y el campo global
}

\section{Rethinking the (rural) space: between the global countryside and the individualization}

\author{
Ángel Paniagua-Mazorra*
}

\begin{abstract}
Usually, the study of the rural category is the core of rural studies. The new rurality in Latin America, or the restructuring and rural change in Europe, are limited theoretical constructions to explain the present complexity of rural spaces and societies. These limitations open a new period founded upon mixed and transversal theoretical argumentations to explain social, spatial and environmental processes. These argumentations introduce, clearly, new approaches in the analysis of the rural category, traditionally associated with binary schemes, linked with more flexible categories bound to environmentalism, place, mobility, identity, ethnicity, with a great analytical potential for transversality and ductility. In this tendency the present contribution suggests a new concept: the global individual field.
\end{abstract}

Keywords: rural theory, space, flexible approaches.

\section{Resumen}

Con frecuencia el análisis de la ruralidad, desde diversas perspectivas, ha constituido el corazón de los estudios rurales. No obstante, la nueva ruralidad en Latinoamérica o la reestructuración y cambio rural en Europa han constituido construcciones teóricas, en las que se ha evidenciado su limitación para explicar de forma global la complejidad de fenómenos desarrollados en áreas rurales. Esto puede estar dando paso a una etapa donde adquieren más peso los marcos teóricos de carácter más general y transversal en la explicación de fenómenos y procesos sociales, espaciales y ambientales. Ello supone, a partir de diversas perspectivas, vencer la idea de lo rural como una categoría fija y rígida, por categorías más flexibles, donde en muchas ocasiones la ruralidad se ve solapada y forzosamente asociada a categorías como ambientalismo, lugar, movilidad, identidad, etnicidad, que se pueden utilizar transversalmente de una manera dúctil. En esta tendencia se propone un nuevo concepto: elcampoindividualglobal.

Palabras clave: teoría rural, espacio, acercamientos flexibles.

* Centro de Ciencias Humanas y Sociales y Consejo Superior de Investigaciones Científicas, España. Correo-e: angel.paniagua@cchs.csic.es. 


\section{Introducción $^{1}$}

Hace pocos años indicamos que los estudios rurales, sobre todo aquellos más ligados a una base geográfica, podrían entrar en una fase de liquidación. Esta afirmación la hicimos con base en el notable número de trabajos sobre áreas y espacios rurales que presentan marcos teóricos y metodologías que se pueden considerar transversales, es decir, se pueden aplicar a cualquier tipo de lugar, sea o no rural o estrictamente rural (Paniagua, 2006). Después, la evolución de los estudios rurales en el ámbito internacional han reforzado esta tendencia. No sólo se ha reforzado la transversalidad, poniendo el acento en lo ambiental, lo cultural, lo poblacional, con metodologías no estrictamente rurales, por ejemplo las derivadas de la incorporación de la sociología cultural al análisis del espacio rural o el análisis de tipo demográfico en el estudio de poblaciones que residen en espacios que se consideran rurales. Además, la reciente evolución de los estudios sobre el lugar ha incrementado los acercamientos que son denominados híbridos o mixtos, como una constante, nicho o campo común ampliamente reconocido en la actualidad en todas las disciplinas sociales, principalmente en la antropología, la sociología o la geografía (Whatmore, 2001; Santos, 2009) y que nosotros abordamos recientemente (Paniagua, 2009, 2010). A lo largo de esta investigación nos ocupamos de un acercamiento más complejo, ligado parcialmente a la tendencia expuesta antes: la coincidencia de procesos de individualización y la generación de un campo global, en relación con otros ejes diferenciados. Consecuencia, en definitiva, por una parte, de aproximaciones que en buena medida exceden notablemente el campo de análisis de la ruralidad, como elemento determinante y vertebrador de los estudios rurales y, por otra, de la aplicación de marcos teóricos generales de la sociología o la propia geografía para explicar el comportamiento de los espacios rurales. Esto tiene una consecuencia clara: la (des)vertebración de la ruralidad como eje de estudio de los espacios rurales. La ruralidad como componente principal o eje de los estudios rurales queda sustituida por otros ejes, que habitualmente son más transversales o que oscilan entre el individuo y la globalidad. A su vez, en cada uno de estos ejes es posible observar una clara oscilación entre lo individual o lo global. Dichos ejes, ligado a la gobernanza (rural), lo ambiental y lo étnico descentrarían el notable peso que hasta ahora recae en el estudio de la ruralidad.

En todo caso, para llegar a este punto es preciso explicar un notable itinerario que arranca en los ańos setenta y que se prolonga hasta la ac-

${ }^{1}$ Este artículo se presentó como ponencia en el VIII Congreso Latinoamericano de Sociología Rural, Porto de Galinhas, 2010, y una primera versión forma parte de sus actas. 
tualidad. Nos detendremos especialmente en el debate conceptual sobre la reestructuración y lo que consideramos su agotamiento, que da lugar a un campo más abierto en los estudios sobre los espacios rurales.

\section{Reestructuración y nueva ruralidad: una mirada desde ambos lados del Atlántico}

Podemos considerar que los estudios rurales se constituyen de una subdisciplina en el ámbito de las ciencias sociales con distintos ritmos en los ańos cincuenta, aunque con una consideración de tipo académico e incluso social relativamente inferior a todas las subdisciplinas ligadas a lo urbano, marco espacial en el que habitualmente se ha admitido una mayor complejidad. Así, los estudios rurales durante mucho tiempo se armaron alrededor de la actividad agraria y el poblamiento tradicional.

A mediados de los años sesenta, y sobre todo a partir de la década de los setenta, se produce un claro giro en los estudios rurales que afecta, en mi opinión, sobre todo a la sociología y la geografía (Paniagua, 2006). Ello coincide, por ejemplo, con la publicación de la famosa obra de Pahl (1965) sobre el urban-rural continuum, así como otras obras relevantes que también promueven un cierto giro en la sociología rural, que vuelven a poner de actualidad el debate, ya (in)interrumpido, sobre las fronteras y relaciones entre el mundo de lo rural y el mundo de lo urbano. Es en esta etapa cuando comienzan a existir claves notables de una mayor complejidad y diversidad social en las zonas rurales. De cualquier manera se presenta una notable imprecisión en las rutas a seguir por los estudios rurales que trataba de reunir tres áreas de trabajo de forma interrelacionada, como el estudio de las transformaciones demográficas y socioeconómicas, el estudio de las zonas de baja densidad y un paisaje campestre. Las orientaciones más sociales quedaban relacionadas esencialmente a las políticas de colonización o instalación de nuevas poblaciones y a la interpretación de los procesos iniciales de cambio y transformación social en zonas consideradas como rurales.

También en la década de los años setenta es posible indicar el surgimiento de los estudios de ruralidad, los cuales de forma sistemática tratan de deslindar las realidades urbanas y rurales. Aquí podríamos plantear varias hojas de ruta desde distintas disciplinas. Así, desde el punto de vista de la sociología se plantea la diferenciación rural-urbana de una forma hasta cierto punto retórica y en forma de debate, mientras que a partir de otras disciplinas -como la geografía- se trataba de sistematizar, deslindar y delimitar lo rural de lo urbano.

Estos intentos de diferenciación se establecen sobre áreas definidas, o incluso sobre países, mediante el uso de las estadísticas oficiales disponi- 
bles y fundadas en metodologías cuantitativas. La puesta en marcha, en esta época, de los índices de ruralidad ha permitido poner de manifiesto que la ruralidad se va moldeando de forma sucesiva con el tiempo, también sobre bases de tipo cuantitativo (Cloke y Edwards, 1986; Harrington y O’Donoghue, 1998). Aquí es donde ya se pueden marcar dos claros itinerarios que se irán entrecruzando y entremezclando, dependiendo en muchas ocasiones del potencial académico de cada disciplina y la adscripción a una u otra corriente de destacadas figuras del moderno pensamiento rural: la vía ligada a la economía política y la vía ligada a lo social, cultural y finalmente ético.

Pero el debate sobre la ruralidad tiene más implicaciones en el desarrollo de los estudios rurales. Así, la plena aplicación de la economía política suponía la integración de una notable especificidad de lo rural en los procesos de cambio de tipo socioeconómico global. Es decir, había unos procesos socioeconómicos rurales globales que cristalizaban localmente. El desarrollo de este supuesto y sus distintas implicaciones sociales y espaciales ha sido un claro eje de los análisis científicos rurales, hasta prácticamente la actualidad, y en nuestra opinión ha condicionado (y limitado) de forma notable el debate sobre lo rural. Desde esta perspectiva los procesos de cambio global adquieren una determinada forma en cada área rural, es decir, se produce una (inter)actuación de procesos globales y estructuras locales, lo que lleva a la configuración de ciertas zonas producto en las que se presenta una inclinación diferenciada ( $\mathrm{Pa}-$ niagua y Hoggart, 2002). Así se podría admitir toda una galería de espacios rurales, desde aquellos más fundados en la conservación hasta otros más asociados a la producción, fruto de la intersección de procesos de tipo global de carácter político, económico, cultural y demográfico (Paniagua y Hoggart, 2002; Murdoch et al., 2003). Implícitamente se acepta que las intersecciones ligadas a unos tipos básicos se traducirían mediante matices infinitos. En este marco se presenta una, podríamos decir, decisiva utilización la obra de Urry $(1981,2000)$. Uno de los ejes de pensamiento de este autor es la translación de los procesos globales en procesos sociales concretos, y más específicamente en el marco de clases sociales, como un elemento de manifiesta importancia en los procesos dinámicos en áreas de características rurales. Se podría considerar que cada área constituye un pequeño mundo social donde se expresan las antiguas y nuevas relaciones de tipo social.

Pero se plantean notables interrogantes en todo esto que se podrían sintetizar en cómo se describen y se plantean los procesos globales en áreas concretas: ¿qué es un proceso global?, ¿cómo se traslada a una concreta?, ¿cuáles son los canales y cómo se articulan entre ellos? Y, por último y más relevante, ¿cuáles son los procesos globales rurales y cómo se carac- 
terizan y particularizan respecto a los urbanos? La dificultad de conceder una adecuada respuesta a todo esto provoca un debate notable, que como luego comprobaremos conduce a un cierto agotamiento de sus planteamientos académicos. También ha provocado un cierto deterioro y desvirtuación en la utilización de la reestructuración rural, convirtiéndola más en una óptica de trabajo (Hoggart y Paniagua, 2001). De esta manera los espacios rurales se conformarían como procesos de tipo complejo (Hoggart y Paniagua, 2001).

Los estudios de base cultural, desde mi punto de vista, constituyen ya claramente una línea alternativa a lo expuesto, lo rural queda más singularizado y queda ligado, en su última evolución, a componentes de tipo ético.

Retomando el último punto, con los estudios de base más cultural se extiende la complejidad en las corrientes analíticas de los estudios rurales. La cultura puede entenderse tanto en términos de evolución interpretativa de las áreas rurales como en términos de una mayor pluralidad de vías o marcos interpretativos. Podríamos indicar que ambas dimensiones se pueden ver como ciertas: existe una evolución de lo funcional a lo cultural y de aquí a lo ético, y también una coexistencia analítica de lo funcional, la economía-política y las corrientes socioculturales (ver también Cloke y Thrift, 1994).

De esta manera, lo cultural rearticula a la vez que enriquece el debate sobre la ruralidad, posibilitando la generación de nuevos marcos y contextos analíticos (Cloke y Little, 1997).

En todo caso sería posible establecer que la polémica sobre la ruralidad en el marco de lo cultural presentas dos caras, una de carácter alternativo respecto al establecimiento de unos límites estrictos y topográficos de lo rural, y otra de alguna manera asociada a la anterior pero fundada en la representación de la ruralidad. Ello conduce a un enriquecimiento temático muy ligado sobre todo a estilos de vida alternativos o a nuevas visiones de la ruralidad. Pero ello también encierra una notable visión plural de la sociedad rural, donde cada grupo social puede generar su propia representación espacial (Phillips, 1998; Paniagua, 2004).

Así, sería posible simbolizar el espacio rural en una progresión desde lo constructivo, pero la reducción de lo espacial a un signo encierra sin embargo notables disensiones en la compresión de la ruralidad (Paniagua y Hoggart, 2002). En efecto, la simbolización supone también simplificación y de igual forma enriquecimiento, dado que cualquier símbolo se puede utilizar de múltiples maneras. Así, la ruralidad carecería de un espacio o contexto predefinido y puede ser notablemente maleable de acuerdo con intereses o contexto diferenciados. 
Dichos contextos se relacionan sobre todo con una desigual composición social que se admite en la base de los estudios culturales, sobre la cual se interpreta y reinterpreta la ruralidad. El desarrollo de metodologías de análisis cualitativo es una de las características más notables de esta nueva etapa, más propicia al interés de la investigación, por su carácter adecuado a áreas reducidas de trabajo y a una investigación más dirigida por el propio investigador (Paniagua, 2004). De esta forma, el análisis cualitativo hace más fluido y flexible el análisis de grupos sociales, e incluso posibilita el desarrollo de estudios a medida de los intereses del investigador. Ello permite la puesta en práctica de estudios rurales de tipo experimental en los cuales el investigador introduce componentes (en forma de espacios, personas, acercamientos) como si dirigiese un laboratorio (Paniagua, 2004). Tres áreas de estudio han sido destacadas, creemos que con una base universal: las interrelaciones entre la naturaleza y el espacio (y sociedad) rural, la marginalidad y la ruralidad, y la ruralidad como objeto de construcción (Little, 1999).

En todo caso, no todo son luces, también existen algunas sombras sobre los estudios de base cultural. Las críticas que se suelen realizar a este tipo de orientaciones están fundadas en: los notables interrogantes para el uso de sus resultados por administraciones públicas u otros agentes de tipo social o económico, su escasa operatividad al margen de la academia y la relativa inexistencia de categorías analíticas globales o generales. Por último, también se advierte la debilidad para realizar grandes interpretaciones de conjunto ligadas a estudios muy detallados e intensivos, pero de base sociogeográfica reducida y notablemente interpretativos (Paniagua, 2004).

Desde Latinoamérica qué panorama se puede trazar en paralelo, al expuesto, que sirva para Europa (o al menos parte de ella) y una parte del mundo anglosajón no europeo. No es fácil plantearlo debido a las desiguales tradiciones académicas, pero nos atreveremos a insistir en los principales paralelismos, escepticismos y perspectivas de futuro, atendiendo para ello a algunas de las principales reflexiones que han aparecido, sin ánimo de ser exhaustivos. En otro sitio escribimos que los estudios rurales, sobre todo los geográficos en Latinoamérica, no presentaban una tendencia homogénea (Paniagua, 2006). Hasta los años setenta dicha tendencia queda ligada a modelos descriptivos de tipo socioespacial. También indicábamos que a partir de esos años los análisis han tratado más sobre las repercusiones de las transformaciones económicas internacionales en los estudios rurales, los complejos de producción agroindustrial y las formas de penetración capitalista en sociedades agrarias tradicionales. Todo ello no exento de numerosos trabajos sobre población rural. Por la fortaleza de tales debates y la tardanza en la articulación de escuelas nacionales, se retrasan en aparecer temas que pudieran establecer un para- 
lelismo con el otro lado del Atlántico: los debates sobre el espacio rural y el papel del Estado, regidos sobre todo por la economía política, y que fructificaban en los efectos de la globalización en áreas rurales de distinto rango. También aparecen como en el otro lado del Atlántico, aunque con un cierto retraso, las aproximaciones cuantitativas al estudio de lo rural (Gutiérrez y González, 2001), no exentas de ciertas aproximaciones sociales (Levi, 1991; Paniagua, 2006). Desde este punto nos vamos a referir a la denominada nueva ruralidad en Latinoamérica. La nueva ruralidad presenta, desde mi punto de vista, algunas características que permiten su comparación con lo que ocurría (ocurre) principalmente en Europa, dado que se pretende generar una argumentación que tenga validez para el conjunto de Latinoamérica. Este marco argumentativo tiene como uno de sus objetos esenciales la diferenciación entre lo rural y lo urbano, o más concretamente de cualificar la ruralidad, a lo largo del continente. Implícitamente creo que busca vertebrar una sola noción de ruralidad para Latinoamérica. Esta ruralidad estaría regida por componentes modernos, en oposición a otra ruralidad tradicional. La ruralidad tradicional estaría regida por una aproximación funcional ligada sobre todo a la agricultura. Los tipos de agricultura y su inserción en el complejo global determinarían en buena medida esa forma tradicional de entender la ruralidad (Llambí, 1997, 2004; Pérez, 2001).

Si la ruralidad (lo rural) es el determinante de la nueva ruralidad, ésta se entiende desde un complejo de relaciones sociales características ligadas a la comunidad rural que se advierte singular en la definición de la nueva ruralidad. El espacio o territorio rural queda determinado por la extensión de dichas relaciones sociales características y no existiría en tal sentido un límite nítido de lo rural, como categoría que singulariza y concede valor al espacio o territorio rural (Gómez, 2008). El espacio rural contendría un conjunto de actividades. Algunos autores, de manera asociada a esta (nueva) diversidad de actividades, establecen una sociedad plural, sin definir quizás totalmente si esta sociedad heterogénea es una de las características de la nueva comunidad rural (Bendini, 2008). Es decir, si la heterogeneidad es una característica intrínseca de la comunidad rural en los espacios latinoamericanos y si ésta se reproduce a través de las nuevas actividades rurales. Como a este respecto plantean Riella y Mascheroni (2008), la nueva ruralidad haría referencia a la existencia de nuevas actividades rurales no agrarias (las viejas). Para tales autores nuevo o viejo no significan transformaciones notables y acusadas, sino que permite resaltar nuevas realidades sociales habitualmente no consideradas. Aquí volvemos a la perspectiva espacial o territorial que en realidad supone un vencimiento del enfoque sectorial agrario. La cualificación de este nuevo espacio o territorio rural no agrario tiene que estar ligado al surgimiento o cristalización 
de nuevas dinámicas sociales. Nuevas actividades, nuevos espacios y nuevas sociedades o microsociedades quedarían implícitamente ligadas desde este punto de vista. En todo caso no se salva el escollo de explicitar qué es todo el gran mundo del empleo o actividad no agrícola. ¿¿Constituye todo ello un solo indicador de nueva ruralidad? En este punto pensamos que la nueva ruralidad latinoamericana presenta algunas similitudes -aunque producidas con posterioridad- con la reestructuración rural diseñada en Europa. También algunas de sus más notables debilidades.

Hasta aquí quizás este enfoque no sea totalmente preciso, creemos que los problemas provienen de dos vías diferentes: una la inserción del plano territorial en el plano social-comunitario, otra el diseño de una ruralidad latinoamericana.

La inserción del plano espacial o territorial en el sociocomunitario creemos, que al igual que en Europa, no logra articular de la mejor manera los procesos de diferenciación espacial más allá de la diferenciación urbano-rural. Es decir, si la especificidad rural se articula de forma diferenciada en distintas ruralidades bajo un mismo eje. Ello llevaría a establecer diferentes relaciones sociocomunitarias que fundamentan espacios rurales diferenciados, entre ellos y en relación con los procesos urbanorurales y los procesos globales-locales que caracterizan y fundamentan los supuestos teóricos de las nuevas ruralidades o reestructuraciones rurales.

Quizás una de las problemáticas de la nueva ruralidad sea principalmente la consecución de un concepto de ruralidad homogéneo para toda Latinoamérica. Esto ha sido reconocido por algunos autores, como Grammont (2008), y a este respecto también podría tener un cierto punto de comparación con el debate de la ruralidad en Europa. Qué es lo característico de la nueva ruralidad para toda Latinoamérica y qué es la especificidad de la nueva ruralidad en cada país. Es decir, en qué puede contribuir lo rural de cada país a una ruralidad latinoamericana (Bendini et al., 2006). En este punto creemos que ha faltado un cierto diseño de los ejes clave de la ruralidad latinoamericana, bien sea a través de los enfoques más sociales-comunitarios o bien mediante enfoques más ligados a la economía política. Pero creemos que el principal punto clave es la insistencia en la ruralidad como eje que articula el debate. Sobre todo tratar de encontrar especificidades de la ruralidad en amplios territorios geográficos, ligados a la cristalización de procesos globales-locales en comunidades rurales y a la generación de espacios nuevos o antiguos rurales. Por ello, y sin que constituya ningún punto de crítica genérica, la nueva ruralidad latinoamericana no se fundamenta en un claro armazón teórico que integre procesos sociopolíticos y socioeconómicos, como se ha pretendido en Europa, sino que se mueva sobre todo en la enunciación de listas de cambios o transformaciones, agrupadas o no, que de alguna 
manera eran -y son- comunes al espacio latinoamericano con mayor o menor intensidad. Ello hace que no aparezca de una manera clara y significativa una conceptualización de la ruralidad latinoamericana, dado que muchas de esas transformaciones con distinta formulación, presentación e intensidad podrían aparecer en otras partes del mundo, incluso en algunos países europeos de modernización tardía.

Aunque de una forma más minoritaria, también enfoques más culturales que tratan de abordar los procesos de transformación rural reproducen algunos de los vicios que se han advertido en Europa, su excesivo carácter micro y su fuerte relación con la realidad ad hoc que estudia. Son claramente pertinentes para el estudio de las asociaciones o fracturas entre el campo y la ciudad, la generación de nuevas actividades y la complicación del panorama con cuestiones de tipo étnico y su entronque en los intereses locales, regionales y nacionales (por ejemplo, Paredes, 2009). Creo que estos enfoques tienen en su haber una más notable caracterización de las relaciones intrínsecas de lo rural, lo que sirve para cualificarla, pero, ¿̨en qué espacio?

Así, en alguna medida -aun con distintos caminos, intensidades y problemáticas- la nueva ruralidad se ha encontrado con algunos problemas parecidos a la reestructuración. Creemos que los problemas de una definición robusta y compleja que se establecía hace una década para la reestructuración (Hoggart y Paniagua, 2001) también se aplican a la nueva ruralidad.

\section{Hacia dónde va el estudio de los espacios rurales: de la individualización al campo global}

En mi opinión, los continuados debates sobre la ruralidad como eje estructurado de los estudios rurales han entrado en una relativa fase de agotamiento, desde ambos lados del Atlántico (Paniagua, 2006). La retórica o el debate circular que no va más allá de la pura semántica han erosionado notablemente dicha discusión, que quedó zanjada en sus términos ligados a la economía política con las contribuciones de Hoggart y Paniagua (2001) y Murdoch et al. (2003) (ver también Woods, 2005). En dichos textos se apuntan y reconocen con claridad las insuficiencias de la economía política para analizar los espacios rurales en términos generales. No es nuestro propósito insistir otra vez en esta polémica, que en términos geográficos ha recibido numerosas contribuciones, simplemente apuntamos algunas ideas sintéticas que sirvan para nuestra argumentación.

En primer lugar, la utilización de la economía política ha traído como consecuencia una cierta uniformización en el análisis del espacio, en aras 
de la explicación de los grandes procesos; de esta forma ha ocultado detalles y singularidades relevantes y ha dejado para después los enfoques micro. En segundo, ha tratado de amalgamar la perspectiva local (en relación con lo global en su vertiente político-económica), la comunidad (como aspecto social) y el lugar (como espacio) generando espacios específicos que reaccionaban de forma homogénea ante procesos globales. Es decir, la economía política en buena medida se basaba en una articulación perfecta entre comunidad, localidad y lugar. En tercer lugar, como se indicó, ha profundizado en el estudio de la ruralidad y, en consecuencia, en la especificidad de procesos socioeconómicos estrictamente rurales, lo que condujo a una notable diferenciación urbano-rural. Todo ello lleva a una cierta limitación, ya no puesta en cuestión, de la economía política en el estudio de las flexibles relaciones de los espacios y sociedades rurales y en la puesta en evidencia del individuo-ciudadano. La misma problemática sería posible argumentarla para Latinoamérica, pero ¿es posible comprender en un solo marco analítico realidades tan dispares como las que van del norte de México hasta el sur de Argentina? Es decir, tratar de abordar todas las dimensiones de la ruralidad sobre una única argumentación compleja supone una notable debilidad cuando se quiere explicar realidades muy complejas o incluso dispares.

Muy recientemente algunos analistas (Akran-Lodhi y Kay, 2010, y autores en los que sustentan) han argumentado que la cuestión agraria, quizás uno de los puntos más notables de la economía política, no se podía describir de forma unitaria debido a las variadas formas y circunstancias en que se producía, coincidiendo múltiples procesos de manera multifacética e incluso contradictoria que reconfiguran las bases de los procesos laborales y económicos de forma fluida y notoriamente compleja. Algunas aportaciones más empíricas parecen corroborar esta línea argumental en el campo de las new politics y la democracia rural, como los trabajos sobre diferentes áreas de Mexico de Fox et al. (2009) y Otero (2004), y en Brasil de Wittman (2009); o en interpretaciones sobre movimientos sociales rurales transnacionales en Latinoamérica (Woods, 2008). Todos ellos ponen énfasis en las complejas y múltiples trayectorias a nivel de ciudadano-individuo agrario-rural en respuesta a la percepción particular de cada individuo en su contexto (Gudeman, 2004). La agregación de respuestas individuales en cada sitio constituiría la respuesta agregada ante diferentes y particulares presentaciones de procesos de reestructuración. Debemos entender que estos procesos tienen una base más cualitativa que cuantitativa, indudablemente por ejemplo en Latinoamérica, los indicadores de pobreza u otros relativos al nivel de vida son más elevados en áreas rurales que en urbanas, pero no es menos cierto, como indica Kay (2009), que sólo a través de vencer la diferenciación del binomio rural-urbana muchos 
individuos y hogares encuentran una salida a sus condiciones de vida (Pérez y Andrade, 2003; Glockner, 2008; Paniagua, 2008), sobre todo en el marco de un reconocido auge de la economía rural no agraria que establece una relación flexible con el mundo urbano y otras esferas transnacionales (López y Valdés, 2000; CEPAL, 2010). Este punto de vista también se sustenta parcialmente en Wood y Roberts (2005: 10-11). Una de las salidas habituales a la pobreza y al acceso a la tierra mediante la reforma agraria, a largo plazo lleva implícito un claro proceso de individualización. Como bien indica Escobar (2010), el concepto de una única y simple idea de modernidad se movería hacia un conjunto de modernidades. Esta argumentación es de notable interés para lo que aquí tratamos, tanto en Europa como en Latinoamérica es posible admitir múltiples trayectorias donde se conjugan ejes, planos y escalas, y donde la realidad se establece en el marco de una permanente inestabilidad. Esto nos aleja mucho de un solo marco explicativo hacia el que puedan converger grandes espacios. Indicado de otra forma, múltiples realidades y expectativas pueden convivir en un único espacio.

A partir que aquí, sobre la base de estudios culturales y lo que yo entiendo como su evolución a los estudios fundados en la ética o individualización, no existe una clara base argumental o teórica en los estudios rurales.

Además, la ampliación de la base de los estudios rurales (Holloway y Kneafey, 2004), dado que los distintos acercamientos se van superponiendo y no eliminando, hace que cada vez se amplíe más la base temática. Este juicio lo establecemos a partir del peso que en la actualidad tienen las investigaciones sobre lo que podríamos convenir que son bordes sociales o espaciales. Los otros han pasado a adquirir un papel más relevante, sobre todo en los estudios de orientación cultural y social, en el marco de un redescubrimiento de los estudios de comunidad.

Desde este punto pretendemos poner el acento en los procesos de individualización que, como hemos apuntado, presentan tres grandes áreas de interés (Paniagua, 2006): en primer lugar una especial atención a los procesos de individualización como expresión o referente adecuado de los análisis rurales; en segundo, una expresión del análisis individualizado del espacio rural, reforzando la relación individuo-espacio (Paniagua, 2010); en tercer lugar, como indicamos (Paniagua, 2006), una unicidad espacial en el análisis posmaterialista de la ruralidad.

Un componente importante de este nuevo panorama es el análisis de las relaciones binarias para el estudio de la ruralidad a través de múltiples planos humanos, institucionales, estructurales, o incluso atributos individuales, y al estudio de las realidades o campos globales no sólo como polo opuesto del individuo, sino como contrapunto (re)articulado de los 
actuales procesos sociales y económicos que se constituyen alrededor del individuo-ciudadano que se mueve sobre un campo que se comporta como un plano cada vez más global. Es decir, el campo se articularía a través de múltiples relaciones donde lo rural-urbano (muchas veces ligado al aspecto local-global), que habitualmente definía la ruralidad, constituye una más y en ciertas y diversas ocasiones no la más relevante. Podríamos indicar que la relación que pasaría a adquirir más relevancia no sería la local-global (que en general caracteriza los estudios rurales), sino la individual-campo global. Con ello se pretende recoger una cierta invitación teórica que, aunque todavía no del todo asentada en los estudios rurales, ya ha sido apuntada en diversos trabajos (Paniagua, 2008, 2010) y que tiene una cierta consistencia en estudios no rurales o urbanos (Hiernaux, 2005). Todo ello también se relaciona con una cierta dilución a la comunidad como eje o referente de pertenencia para quedar sustituido por el individuo, que se puede mover por el espacio rural-rural, ruralurbano o urbano-rural, todo ello en esferas regionales, nacionales o transnacionales, y que establece relaciones de identidad directa con el espacio y no sólo con el entorno. Parcialmente este punto de vista ha sido sustentado por Kay (2009) cuando observa que en la época de la globalización la interacción y fluidez entre lo rural y lo urbano se ha incrementado notablemente, en términos de capital, bienes y trabajo. De esta forma, muchos hogares rurales pueden construir sus vidas en diferentes sitios más allá de la división urbano-rural, agrario-no agrario e incluso más allá de las fronteras nacionales. Este último punto de vista ya lo sustentamos en Paniagua (2008) en relación con los nuevos contornos móviles y fluidos de las comunidades y hogares rurales, asociados a procesos de individualización.

En este punto creo que debemos detenernos otra vez en la comunidad rural, antes indicamos que la comunidad rural coincide con la localidad y el lugar desde la perspectiva de la economía política, ello ha hecho que los procesos de reestructuración se determinen en el ámbito local o comunidad, como un ente homogéneo, en general la propia comunidad rural se advierte como un agregado que culturalmente presenta respuestas comunes y homogéneas, a lo sumo se llegan a explicitar diferencias -de forma excesivamente simplista- entre élites y el resto de la comunidad. Esto ha provocado que la comunidad se analice como un conjunto que tiene perspectivas y opciones parecidas, no sólo ante cuestiones colectivas sino ante aspectos de índole más personal. Por ello este tipo de visiones de la comunidad se podrían revisar desde la perspectiva de los individuos, a partir de una visión más moral, en la que diferentes individuos de una misma colectividad-comunidad podrían tener distintas visiones de diversos procesos y adoptar procesos más individualizados en la toma de deci- 
sión. Esto supone de alguna forma conceder más poder al individuo en las relaciones de poder, en la relación con el entorno y en las relaciones intracomunitarias. Pero no supone vencer la comunidad en sí misma, sino reequilibrarla como colectividad o plano mediador, como agrupación de identidades, influencias de poder y relación con el espacio, y en relación con esos procesos globales.

Algunos ejemplos que citamos antes parecen apuntar en esta dirección. Se podría admitir que existen distintas identidades colectivas o comunitarias, de mayor o menor relevancia, que realizan diferentes asociaciones con lo local y el lugar, y una serie de vidas morales que identifican distintos intereses, perspectivas e incluso estilos de vida de individuos. Todo ello en forma de equilibrio permanente e inestable. Ello no disminuye la capacidad de respuesta respecto a procesos globales -habitualmente en forma de redes de solidaridad- que en general se ha asignado a la comunidad, la refuerza, al democratizarla y dotarla de una dimensión flexible y fluida, en relación con su esfera local (sociopolítica) y relativa al lugar (entorno y gestión del espacio), tanto respecto a otras comunidades como en relación con el Estado o los procesos transnacionales.

Pero para insertar adecuadamente la identidad individual o la identidad individuo en el campo global, que en principio podríamos denominar campoindividualglobal, es preciso hacerlo en diversos ejes que sustituyan al global-local que hasta ahora ha jugado un papel relevante en la caracterización de los estudios de comunidad o localidad rural. Para conseguirlo, esta relación se establecería a través de diversos ejes que no adoptarían una relación de binomio como es habitual en los estudios de ruralidad. Todo ello contribuiría a descentrar la ruralidad como eje específico de estudio, lo que permite articular, de mejor manera, las diferentes realidades que se establecen en espacios rurales en Latinoamérica o en la propia Europa.

Las relaciones o ejes relevantes para analizar los espacios rurales serían:

Eje étnico: con frecuencia en la literatura se define como la incorporación de la sociedad multicultural y también cosmopolita en la descripción de la ruralidad. Así, la introducción de los puntos de vista normalmente considerados como minoritarios, como la introducción de puntos de vista indígenas, contribuye notablemente a enriquecer la propia identidad de la vida rural, de sus antiguos estereotipos y de sus modernas identidades. Además contribuye de forma significativa a la conceptualización del espacio, en algunos casos es decisivo en su caracterización. Pero en este sentido también contribuye a distanciar una idea o noción nacional de lo rural por otra más heterogénea. Permite, por otra parte, reenfocar la reconocida y global tendencia a que cada individuo tenga un campo idealizado. Además el eje étnico permite incorporar en muchas realidades, de 
forma analítica, la relación individuo-naturaleza y su incidencia en el uso de los recursos.

Pero el eje étnico también permite considerar uno de los aspectos más relevantes de diferenciación comunitaria en muchas áreas rurales, regidas por complejas y multiétnicas situaciones que combinan diversas identidades y jerarquías, que en muchas ocasiones quedan ligadas a individuos. Si consideramos la ruralidad como un agregado de múltiples identidades, donde situar al individuo ciudadano y cosmopolita, el eje étnico permite incluir una nueva dimensión de hibridación en la consideración de la ruralidad y es un claro punto de replanteamiento de las relaciones selflocals (community)-others o de las más usuales locals-others (Paniagua, 2010). Por otra parte, también permite situar la consideración de la ruralidad y de la individualidad a través de la etnicidad, cuando se establecen derechos étnicos ligados a un determinado espacio, que supone su exclusividad y originalidad respecto al campo global. En todo caso, adoptar como referencia al individuo permite abordar como concepto reflexivo la colectividad, como agrupación de individuos.

Eje ambiental, es en sí mismo multidimensional pero sería posible hacer prevalecer dos planteamientos: por una parte la relación sociedadnaturaleza o la relación naturaleza-cultura (en sí planteamientos en términos de binomio), y por otra, el idilio rural -la atracción idealizada del campo por poblaciones urbanas- sobre el que nosotros hemos insistido en numerosos escritos (Paniagua, 2006). En sí mismo es un eje muy complejo en el que es necesario avanzar, pero que en definitiva remite a representaciones individuales o agregación de identidades individuales en la consideración de la ruralidad, que está en la misma base de la movilidad en las zonas rurales o en la retrospección desde una perspectiva de derechos individuales en la gestión ambiental, en el contexto de renovadas relaciones sociedad-naturaleza. Todo ello afectaría de forma jerarquizada y notablemente desigual a la interacción individuo-campo global en su intermediación comunidad-espacio.

Eje recreativo- turístico que está ligado al establecimiento de amenidades (rurales), a una renovada gestión del patrimonio cultural rural material e inmaterial y a nuevas actividades recreativo-turísticas que contribuyen en su conjunto a desligar de forma desigual, según las zonas, a poblaciones de su labor agraria, pero que a la vez permiten atraer individuos dispares y cosmopolitas a un (desde un) campo global. Se podrían establecer diversas dinámicas que aparecen de forma diferenciada, podríamos sugerir: 1) la migración nacional o internacional ligada a la existencia de amenidades, de un patrimonio cultural y desarrollo de actividades recreativas, todo lo cual supone un incremento de representaciones globales en un campo global, pero también la existencia de un mercado 
global ligado a un campo global. No sería inusual encontrar atractivos en un área lejana, incluso transoceánica, y tampoco encontrar un mercado accesible, también de carácter transoceánico. Muchas de las áreas más características de nuestro campo responden a este tipo y ponen en evidencia la dificultad de confinar representaciones e incluso mercados a un espacio nacional o aun continental. Todo ello supone una notable transformación de las comunidades rurales y constituye un factor de diferenciación interna, también en su simbolismo, y promueve claramente su individualización ligada a derechos, representaciones o intereses no o extra comunitarios; 2) la creación de amenidades y renovación en la gestión patrimonial y establecimiento de nuevos sitios turísticos de base difusa que promueven una diferenciación laboral, y que en muchas ocasiones suponen una transformación de su función original: el cambio de pueblos agrícolas, la transformación de haciendas agrarias o la reutilización de vías tradicionales de comunicación -como los senderos- para nuevos usos deportivos (el senderismo), todo ello promueve una nueva producción de los sitios, una rearticulación del patrimonio material, incluido el paisaje, en realidades renovadas; 3) la revitalización de áreas consideradas rurales en identidades lejanas, a menudo asociadas a élites sociales y la depreciación de otras identidades rurales más regionales o nacionales.

Todo ello, en cierta forma, está ligado a una cierta transformación, sobradamente reconocida de una visión de producción a una idea de consumo en los espacios rurales, que no se produce de manera uniforme espacialmente, pero que dota al individuo o a agrupaciones de individuos de nuevas prácticas económicas y sociales de manera formal o informal. Asimismo, el patrimonio rural adquiere un nuevo valor y (re)conforma nuevos espacios tipo, idealizados, con una nueva carga simbólica para los propios habitantes de las comunidades, así como para las personas externas a las mismas. Todo ello reconfigura necesariamente la relación localcomunidad-lugar, al alterarse los componentes de esta relación y su significado e incrementarse los procesos sociales, culturales y económicos de flexibilización.

Eje ligado al nuevo gobierno rural, diferenciado de un gobierno agrario tradicional (Kay, 2004), donde el foco de interés no es la tradicional explotación agraria, sino el individuo que vive en un espacio rural. Individuo ligado a la concepción de ciudadanía. De esta forma, en el medio rural no sólo se es objeto de políticas como productor sino como residente, como ciudadano. Esto tiene notorias implicaciones sobre la comunidad, su constitución y su inserción, así como en la consideración de los otros. Pero sobre todo dota al individuo de una cierta autonomía o singularidad y lo desliga de una cierta opción de desarrollo, que aunque evidente en áreas con concentración de pobreza, también ha contribuido a minusva- 
lorar al individuo (ciudadano) rural respecto al (ciudadano) individuo urbano. Esto supone superar algunos de los problemas que habitualmente se han puesto de manifiesto en los gobiernos rurales, uno de ellos es la excesiva elitización, esto es, la concentración de poder en ciertas élites de la comunidad, excluyendo a otros grupos sociales o individuos, lo que a menudo provoca una segmentación social, en perjuicio de los grupos e individuos más marginados o necesitados de la comunidad, lo que supone su exclusión de cualquier iniciativa. Esto supondría un incremento de legitimidad, como se ha puesto de manifiesto en algunas experiencias puestas de relieve anteriormente. Este nuevo gobierno rural tendría también un polo de atención a la interfaz intereses del Estado- intereses locales, que en muchas ocasiones se han caracterizado de manera conflictiva y que han generado una reconsideración del poder del Estado local, dentro y fuera de la política rural, desde el gobierno a la gobernanza, donde la comunidad identifica renovados problemas, estrangulamientos, pero también soluciones (Rose, 1996). Ello reconfigura de manera flexible las relaciones de poder en los gobiernos locales. Este proceso, a su vez, también facilitaría la propia gobernanza de las comunidades -incluidos los procesos de desarrollo endógeno-y la construcción de nuevas alianzas. Esto podría generar una gestión renovada por los individuos que componen las poblaciones locales mediante la relocalización de poderes en el contexto de las comunidades. Como consecuencia se produciría una menor marginación política de las áreas rurales y un mayor equilibrio entre actores individuales respecto a aproximaciones de tipo estructural e institucional. La gobernanza local sería un mediador entre los actores individuales y el contexto de mayor o menor amplitud. Los actores individuales serían transformados o moldeados en relación con otros.

\section{Conclusión}

En esta investigación hemos realizado un recorrido por los notables trabajos que han adoptado la ruralidad como eje común de estudio, para poner de manifiesto sus limitaciones como marco explicativo en la actualidad. A veces incluso como algo limitante. Para ello proponemos de forma inicial un nuevo concepto: elcampoindividualglobal.

En todo caso, la presente aportación no supone algo totalmente alternativo al papel de la comunidad rural como algo cerrado entre sociedad, naturaleza y espacio, regido en buena medida por la perspectiva localglobal, sino como una ampliación de la perspectiva de estudio, con dos nuevos extremos: el individuo y el campo global que se pueden encontrar mediados o no por la comunidad, la localidad o el lugar. Pero que en definitiva supone una aceptación del individuo como jugador activo y recep- 
tor pasivo en un campo de juego global, en diversos ejes entre los que hemos propuesto de forma inicial el étnico, el ambiental, el recreativoturístico y el referido al gobierno (rural). A través de los mismos queremos evidenciar la diferente posición del individuo (rural) en distintos contextos que han sido objeto de atención por los analistas de los procesos de cambio y nueva ruralidad. Con los mismos hemos querido amalgamar las posiciones críticas, habitualmente ligadas a los otros en distintos planos, que en su conjunto proyectan una vía de integración de todos los individuos que viven en un determinado sitio. Por ello y para adoptar una posición micro, basada en la distinción, el matiz y la fluidez que dominan la vida cotidiana de los individuos, que creo asimismo que puede resolver posiciones de (in)justicia social, ambiental y territorial, se deben sustentar sobre todo en una visión moral de las mismas. No cabe duda que desde estas posiciones de análisis fundadas en el individuo pueden ser criticadas por todas las limitaciones cotidianas, quizás la principal sea la ausencia de una base material para el sustento digno, y por los condicionantes de las estructuras institucionales y sociales. A menudo detrás de las grandes cifras que sustentan una visión parcial de tal o cual fenómeno (rural), incluida la pobreza, es posible y natural reflexionar sobre la multiplicidad de situaciones, autopercepciones y visiones que es posible encontrar en una población (rural) cada vez menos agraria y lo difícil que es explicarlas a través de la condición (estática y fija) de ruralidad.

En consecuencia, estos ejes que proponemos no tendrían una consideración de binomio como ha sido habitual en los estudios rurales, sino que supondrían planos del juego del individuo en el campo global. Con todo ello pretendemos, por una parte, articular las posibilidades de los enfoques éticos y morales y los procesos de modernidad y, por otra, tratar de conceder un paraguas teórico común a los nuevos procesos rurales que surgen en diferentes áreas geográficas. No queremos con ello radicalizar nuestro enfoque y considerar al individuo como un player que se mueve con libertad sobre el espacio (y en consecuencia sin dificultad). No, en absoluto. Entendemos que existen estructuras rígidas que han prevalecido en los estudios rurales, lugar (territorio) y comunidad serían dos de ellas, también el gobierno agrario (y en cierta medida el rural), pero que simplemente serían condicionantes, no limitantes, en la nueva relación individuo-campo global.

El autor quiere dejar constancia de su agradecimiento al Ministerio de Ciencia e Innovación de España, por el apoyo al proyecto (Código, CSO2008-00953). Asimismo expresa su reconocimiento por los comentarios y reflexiones de dos evaluadores anónimos a una primera versión del presente texto. 


\section{Bibliografía}

Akran-Lodhi, Haroon y Cristóbal Kay (2010), "Surveying the agrarian question: current debates and beyond" (part 2), The Journal of Peasant Studies, 37, 2, Routledge, Londres, pp. 255-284.

Bendini, Mónica, Josefa Salete Barbosa Cavalcanti y Sara María Lara Flores (2006), "Una mirada sobre el campo de la sociología rural en América Latina", en Enrique de la Garza Toledo (coord.), Tratado latinoamericano de sociología, Anthropos-Universidad Autónoma Metropolitana, Barcelona, pp. 247-263.

Bendini, Mónica (2008), "Actores sociales y reestructuraciones en los ámbitos rurales y agrarios de América Latina”, en Edelmira Pérez, María Adelaida Farah y Hubert Grammont (eds.), La nueva ruralidad en América Latina. Avances teóricos y evidencias empiricas, Clacso-Pontifica Universidad Javeriana, Bogotá, pp.133-150.

CEPAl (Comisión Económica para América Latina y el Caribe) (2010), Anuario estadistico de America Latina y Caribe, Cepal, Santiago.

Cloke, Paul y George Edwards (1986), "Rurality in England and Wales 1981: A replication of the 1971 index”, Regional Studies, 20 (4), Routledge, Londres, pp. 289-306.

Cloke, Paul y Nigel Thrift (1994), "Introduction: refiguring the 'Rural'”, en Paul Cloke et al., Writing the rural: Five cultural geographies, Paul Chapman Pub., Londres, pp. 1-6.

Cloke, Paul y Joe Little (eds.) (1997), Contested Countryside cultures: Otherness, marginalisation and rurality, Routledge, Londres.

Escobar, Arturo (2010) "Latin America at a crossroads", Cultural Studies, 24 (1), Routledge, Londres, pp. 1-65.

Fox, Jonathan, Carlos García Jiménez y Libby Haight (2009), "Rural democratisation in Mexico's deep south: grassroots right-to-know campaigns in Guerrero", The Journal of Peasant Studies, 36 (2), Routledge, Londres, pp. 271-298. 
Glockner Fagetti, Valentina (2008), De la montaña a la frontera: identidad, representaciones sociales y migración de los niños mixtecos de Guerrero, El Colegio de Michoacán, Zamora.

Gómez, Sergio (2008), "Nueva ruralidad. Fundamentos teóricos y necesidad de avances empíricos", en Edelmira Pérez, María Adelaida Farah y Hubert Grammont (eds.), La nueva ruralidad en América Latina. Avances teóricos y evidencias empiricas, Clacso-Pontificia Universidad Javeriana, Bogotá, pp. 45-78.

Grammont, Hubert (2008) "El concepto de nueva ruralidad", en Edelmira Pérez, María Adelaida Farah y Hubert Grammont (eds.), $L a$ nueva ruralidad en América Latina. Avances teóricos y evidencias empiricas, Clacso-Pontificia Universidad Javeriana, Bogotá, pp. 23-44.

Gudeman, Stephen (2004), Relationships, residence and the individual: a rural Panamanian community, Routledge, Londres.

Gutiérrez de MacGregor, María Teresa y José González (2001), “Propuesta para fijar diez mil habitantes como límite de una localidad urbana”, Investigaciones Geográficas, 44, Universidad Nacional Autónoma de México, México, pp. 103-118.

Harrington, Vicki y Dan O'Donoghue (1998), "Rurality in England and Wales 1991: A replication and extension of the 1981 rurality index", Sociologia Ruralis, 38 (2), Blackwell, Londres, pp. 178203.

Hiernaux-Nicolas, David (2005), “¿Identidades móviles o movilidad sin identidad? El individuo moderno en transformación”, Revista de Geografía de Norte Grande, 34, Universidad Católica de Chile, Santiago, pp. 5-17.

Hoggart, Keith y Ángel Paniagua (2001), "What rural restructuring?”, Journal of Rural Studies, 17, Elsevier, Amsterdam, pp. 41-62.

Holloway, Lewis y Moya Kneafsey (2004), "Geographies of rural cultures and societies: introduction”, en Lewis Holloway y Moya Kneafsey, Geographies of rural cultures and societies, Ashgate, Londres, pp. $1-14$. 
Kay, Cristóbal (2004) "Las políticas agrarias en Europa y América Latina", en Edelmira Pérez Correa y María Adelaida Farah (comps.), Desarrollo rural y nueva ruralidad en América Latina y la Unión Europea, Pontificia Universidad Javeriana, Bogotá, pp. 81-90.

Kay, Cristóbal (2009), "Development strategies and rural development: exploring synergies, eradicating poverty", The Journal of Peasant Studies, 36 (1), Routledge, Londres, pp. 103-138.

Levi, Sebastian (1991), "Rural change and circular migration to the United States. A case study from Michoacán, México", Investigaciones Geográficas, 23, Universidad Nacional Autónoma de México, México, pp. 33-52.

Little, Joe (1999), "Otherness, representation and the cultural construction of rurality", Progress in Human Geography, 23-3, Sage, Londres, pp. 437-442.

López, Ramón y Alberto Valdés (eds.) (2000), Rural poverty in Latin America, MacMillan Press, Londres.

Llambí, Luis (1997) “Reestructuraciones rurales y globalización. El debate entre economía política y postmodernismo en la sociología rural", Agricultura y Sociedad, 84, Ministerio de Agricultura, Madrid, pp. 325-346.

Llambí, Luis (2004), "Nueva ruralidad y multifuncionalidad de los espacios rurales", en Edelmira Pérez Correa y María Adelaida Farah (comps.), Desarrollo rural y nueva ruralidad en América Latina y la Unión Europea, Pontificia Universidad Javeriana, Bogotá, pp. 91-101.

Murdoch, John, Phillip Lowe, Terry Marsden y Neil Ward (2003), The differentiated countryside, Routledge, Londres.

Otero, Gerardo (2004), ¿Adiós al campesinado?: democracia y formación politica de las clases en el México rural, Universidad Autónoma de Zacatecas, México.

Pahl, Robert (1965), "Urbs in rure", London School of Economics, Londres, mimeo. 
Paredes-Ramírez, Wilfredo (2009), La fractura campo-ciudad y los montubios. (Desencuentros con la ruralidad e indiferencia hacia los montubios), Banco Central de Ecuador-Archivo Histórico de Guayas, Guayaquil.

Paniagua, Ángel (2004), “La geografía rural: entre el peso de la regulación y las orientaciones constructivistas", Document's d'Analisi Geográfica, 43, Universidad Autónoma de Barcelona, Bellaterra, pp. 123-134.

Paniagua, Ángel, (2006), "Geografía rural”, en David Hieraux y Alicia Lindón (dirs.), Tratado de geografía humana, Antrophos, Barcelona, pp. 70-83.

Paniagua, Ángel (2008), “La individualización del mundo rural. Dimensiones analíticas para ¿un concepto generalizable?”, Economía, Sociedad y Territorio, vIII (27), El Colegio Mexiquense, A.C., Zinacantepec, pp. 639-659.

Paniagua, Ángel (2009), “The politics of place: official, intermediate and community discourses in depopulated rural areas of Central Spain. The case of the Riaza river valley (Segovia, Spain)", Journal of Rural Studies, 25 (2), Elsevier, Amsterdam, pp. 207-216.

Paniagua, Ángel (2010), "I live out in the countryside. Exploring extreme processes of individualization in rural Spain", Scottish Geographical Journal, 126 (1), Routledge, Londres, pp. 9-23.

Paniagua, Ángel y Keith Hoggart (2002), "Lo rural: hechos, discursos o representaciones. Una perspectiva geográfica de un debate clásico”, Información Comercial Española, 803, Ministerio de Economía, Madrid, pp. 61-72.

Pérez, Edelmira (2001), "Hacia una visión de lo rural”, en Norma Giarracca (ed.), ¿Una nueva ruralidad en América Latina?, Clacso, Buenos Aires, pp. 17-30.

Pérez-Sainz, Juan Pablo y Katharine Andrade Eekhoff (2003), Communities in globalization: the invisible Mayan Nahual, Rowman and Littlefield, Oxford. 
Phillips, Martin (1998), "The restructuring of social imaginations in rural Geography”, Journal of Rural Studies, 14 (2), Elsevier, Amsterdam, pp. 121-153.

Riella, Alberto y Paola Mascheroni (2008), "Evidencias empíricas sobre la pertenencia de la nueva ruralidad en Uruguay", en Edelmira Pérez, María Adelaida Farah y Hubert Grammont (eds.), La nueva ruralidad en América Latina. Avances teóricos y evidencias empiricas, Clacso-Pontificia Universidad Javeriana, Bogotá, pp. 151-157.

Rose, Nikolas (1996), "The death of the social? Refiguring the territory of government", Economy and Society, 25, Routledge, Londres, pp. 327-356.

Santos-Granero, Fernando (2009), "Hybrid bodyscapes. A visual history of Yanesha Patterns of Cultural change", Current Antropology, 50 (4), University of Chicago Press, Chicago, pp. 477-512.

Urry, John (1981), "Localities, regions and social class", International Journal of Urban and Regional Research, 5, Wiley, Londres, pp. 455-473.

Urry, John (2000), Sociology beyond Societies. Mobilities for the twenty-first century, Routledge, Londres.

Whatmore, Sara (2001), "Hybrid geographies: rethinking the 'Human' in Human Geography", en Doreen Massey, John Allen y Phil Sarre (eds.), Human Geography today, Polity Press, Cambridge, pp. 22-39.

Wittman, Hannah (2009), "Reworking the metabolic rift: La Vía Campesina, agrarian citizenship, and food sovereignty", The Journal of Peasant Studies, 36 (4), Routledge, Londres, pp. 805826.

Wood, Charles y Bryan Roberts (eds.) (2005), Rethinking development in Latin America, Pennsylvania University Press, Pennsylvania.

Woods, Michael (2005), Rural Geography, Sage, Londres. 
Woods, Michael (2008), "Social movements and rural politics", Journal of Rural Studies, 24, Elsevier, Amsterdam, pp. 129-137.

Recibido: 5 de octubre de 2010. Reenviado: 7 de marzo de 2011. Aceptado: 12 de mayo de 2011.

Ángel Paniagua. En 1990 obtuvo el doctorado en geografía humana por la Universidad Autónoma de Madrid. Actualmente es investigador científico en el Consejo Superior de Investigaciones Científicas, Madrid. Es el primer autor español con publicaciones en revistas como Journal of Rural Studies o Land Use Policy. Evaluador externo de más de 20 revistas científicas especializadas en todo el mundo. Autor de más de 100 publicaciones en las principales revistas internacionales y nacionales, esencialmente sobre aspectos ambientales y sociales de las áreas rurales. Sus líneas de investigación se centran en cuatro áreas de interés: 1) procesos de cambio social, ambiental y cultural en áreas despobladas, 2) investigación socioambiental cualitativa, 3) la relevancia de la sociología política y cultural en el reciente análisis espacial y 4) las relaciones entre sociedad y naturaleza. Sus últimas dos publicaciones relevantes son: "I live out in the countryside. Exploring extreme processes of individualization in rural Spain”, Scottish Geographical Journal, 126 (1), Routledge, Londres, pp. 9-23 (2010); "The politics of place: official, intermediate and community discourses in depopulated rural areas of Central Spain. The case of the Riaza river valley (Segovia, Spain)", Journal of Rural Studies, 25 (2), Elsevier, Amsterdam, pp. 207-216 (2009-online 8 enero). Primer artículo publicado on line en el 25 aniversario del Journal of Rural Studies, 25 years of excellence in rural studies'. 